\title{
EFFECTS ON SPERMATOGENESIS IN GUINEA-PIGS, RABBITS AND SHEEP AFTER THEIR IMMUNIZATION WITH SEXUAL ORGAN FLUIDS OF BULLS*
}

\author{
J. MATOUŠKK \\ Laboratory of Physiology and Genetics of Animals, Czechoslovak Academy of Sciences, \\ Liberchov, Czechoslovakia
}

(Received 9th April 1968, revised 4th Fune 1968)

Summary. The effect on spermatogenesis of intratesticular and subcutaneous immunization with genital tract fluids of bulls was studied in sexually mature guinea-pigs, rabbits and sheep.

Immunization with bovine seminal vesicle fluid produced a decrease in the index weight of the testes, a disturbance of spermatogenesis and, in more severe cases, a distortion and contraction of the seminiferous tubules. In all animals immunized subcutaneously, identical circulating antibodies were found in animals with damaged testes and in animals with normal testes. They did not cause spermagglutination, immobilization or spermatotoxic reactions. No circulating antibodies could be found in guinea-pigs and rabbits immunized by a single intratesticular injection.

\section{INTRODUCTION}

The idea that some accessory sexual gland fluids of animals may have aspermatogenic effects first occurred following the observation of azoospermia in a bull immunized with boar seminal vesicle fluid (Matoušek, Dostál \& Fulka, 1966). Results from preliminary hetero-immunization of other species with bovine seminal vesicle fluid confirmed this supposition (Matoušek, 1966).

Further study was therefore directed to the aspermatogenic effects of all bovine accessory sexual gland fluids, including testicular fluid and blood serum. Results obtained from this study are published in the present paper.

\section{MATERIALS AND METHODS}

Collection of fluids

Genital glands were obtained from sexually mature bulls killed at the slaughterhouse. The genital tract fluids were obtained as soon as possible after the animal had been killed.

Fluids were gently expressed from the ampullae and seminal vesicles. Epididymal fluids were collected in centrifuge tubes from the cauda epididymidis. An incision was made in the posterior part of the cauda epididymidis,

\footnotetext{
* This paper reports on part of a project investigated within the International Biological Programme.
} 
a hypodermic syringe was inserted into the epididymal ducts and the fluids were expelled by air. Testicular fluids were obtained by careful incision of the surface of the gland with a scalpel after removal of the tunica albuginea. The fluid exuding from the cut surface was collected into a test tube.

After collection, the fluids were immediately centrifuged for $20 \mathrm{~min}$ at $8000 \mathrm{rev} / \mathrm{min}$ and stored in the refrigerator at $-20^{\circ} \mathrm{C}$.

\section{Immunization and the detection of antibodies}

Guinea-pigs and rabbits were immunized by a single intratesticular injection of 0.2 and $0.3 \mathrm{ml}$ fluid, respectively. The animals were castrated and blood was collected from them 35 days after the injection.

Other injection routes used to induce immunization were subcutaneous, intramuscular, or a combination of both. Fluids were injected into the neck region once weekly for 4 weeks, the amounts, per injection, being $0.2 \mathrm{ml}$ for guinea-pigs, $0.5 \mathrm{ml}$ for rabbits, $5 \mathrm{ml}$ for rams and boars, and $10 \mathrm{ml}$ for bulls. Castration, blood collection and slaughter of the animals took place 7 to 10 days after the last injection. Secondary immunizations were not performed until at least 3 to 4 months after the primary immunization. All immunizations were carried out with a mixture of fluids obtained from several animals.

The character of the antibodies formed in the immunized animals was studied by means of sperm-agglutination and immobilization (Quinlivan, 1966), the spermatotoxic test using cyanin B to differentiate damaged from intact spermatozoa (Fulka, Valenta, Pavlok \& Icha, 1960), the complementfixation test (Kwapinski, 1965), the agar-gel double diffusion technique ( $1 \%$ agar-gel with borate buffer) and immuno-electrophoresis (Grabar \& Burtin, 1964). In addition, the active local anaphylaxis test (Campbell, Garvey, Cremer \& Sussdorf, 1963) was used for animals which had received a single intratesticular injection. Ejaculated spermatozoa from bulls and rams and epididymal spermatozoa from rabbits were used for agglutination, immobilization and spermatotoxic tests.

\section{Effects of immunization on spermatogenesis}

The testes of immunized males were weighed and examined histologically after staining with haematoxylin and eosin. The index weight, i.e. testis weight in $\mathrm{g}$ or $\mathrm{kg} \times 1000 /$ body weight in $\mathrm{g}$ or $\mathrm{kg}$, was used to express testis weight. The extent of the damage to the testes was evaluated according to the loss of spermatogenic cells, the decrease in diameter of the seminiferous tubules, and their degree of distortion or disappearance. The degree of testicular damage was assessed on a scale from 0 (normal histological appearance) to 4 (disappearance of all cells of the spermatogenic layers and of the convoluted tubules). The mean number of convoluted tubules was calculated by measuring 150 to 200 tubules from different parts of two testis samples. In rams, ejaculates were analysed and the size of the testes was measured.

Binding or blocking of the aspermatogenic factor

Seminal vesicle fluid, previously absorbed by once-washed spermatozoa in a volume ratio of $1: 1$, was used for immunization in order to study whether the 
aspermatogenic factor of bull seminal vesicles would become attached to homologous ampullar or epididymal spermatozoa.

The possibility of blocking the aspermatogenic factor of bull seminal vesicles was also studied by making a 1:1 mixture of immune antibodies obtained from animals with damaged testes and bull seminal vesicle fluids. After half an hour, the mixture was centrifuged for $20 \mathrm{~min}$ at $8000 \mathrm{rev} / \mathrm{min}$ and the supernatant was used for immunizing animals of the species which had supplied the immune antisera. Immunization doses were increased by $50 \%$ compared to the use of seminal vesicle fluids only. Normal sera were treated in a similar manner.

\section{RESULTS}

Degenerative changes in testes after single intratesticular injections

Considerable degenerative changes and aspermatogenesis were found in the injected testis of guinea-pigs and rabbits following the administration of the seminal vesicle fluid of bulls (SVFB). The injection of the ampullar fluid of sexually mature bulls (AFB) into guinea-pigs also had the same effect. Other fluids had practically no effect (Table 1).

In the majority of animals, only the injected testis showed degenerative changes while the other remained unaffected. In only one out of ten guineapigs treated with SVFB was the second testis also damaged. Two of ten guineapigs injected with AFB sustained damage to the uninjected testis as judged by the index weight of the testes and histological analysis.

SVFB absorbed by ampullar spermatozoa also caused aspermatogenesis and degenerative changes (Table 1). The changes following intratesticular injection of SVFB were usually irreversible.

\section{Degenerative changes in testes after subcutaneous injections}

Subcutaneous (s.c.) injection of SVFB was followed in some animals by a significant decrease in the index weight of the testes (i.e. compared with controls) and aspermatogenesis (Table 2). Damage was not alleviated when SVFB was absorbed by ampullar spermatozoa (Table 2 ).

In rams, the damage to the testes was of a temporary, reversible character. A single exception to this occurred in the case of one testis in one ram. From observations of four guinea-pigs and two rabbits, it appeared that the damage caused by s.c. injections of SVFB was in most cases only temporary.

Secondary immunization very rarely caused new damage to testes which had returned to normal.

The experiment designed to block the substance provoking immunization by means of specific antisera was unsuccessful. The mixture of SVFB and antisera, when injected into the left testis of four guinea-pigs, caused a decrease in the index weight of the injected testes to $0.32 \pm 0 \cdot 10$ and histological damage of the third to fourth degree. One of two rams immunized s.c. exhibited a decrease of $26 \%$ in testicular size, and the ejaculate was azoospermic for 42 days.

Fluid from the ampullae, the cauda epididymidis and the testes, and the blood serum of bulls injected s.c. for 4 weeks into six guinea-pigs did not cause 


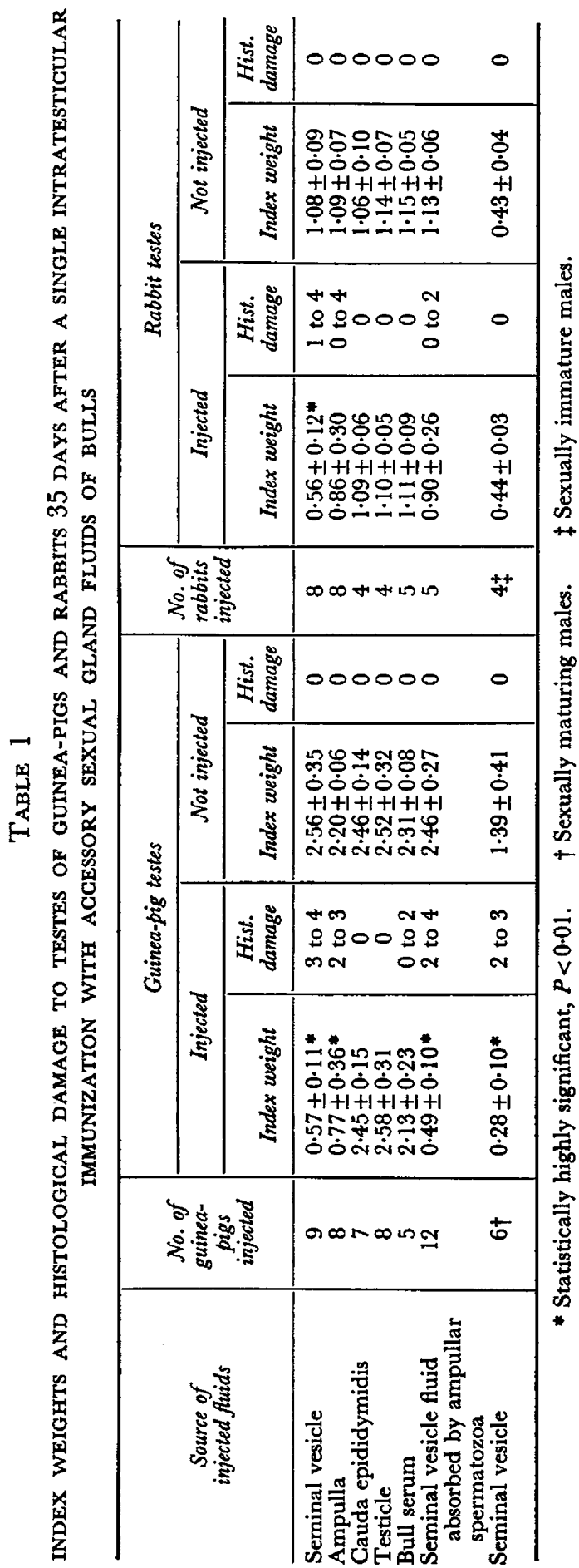




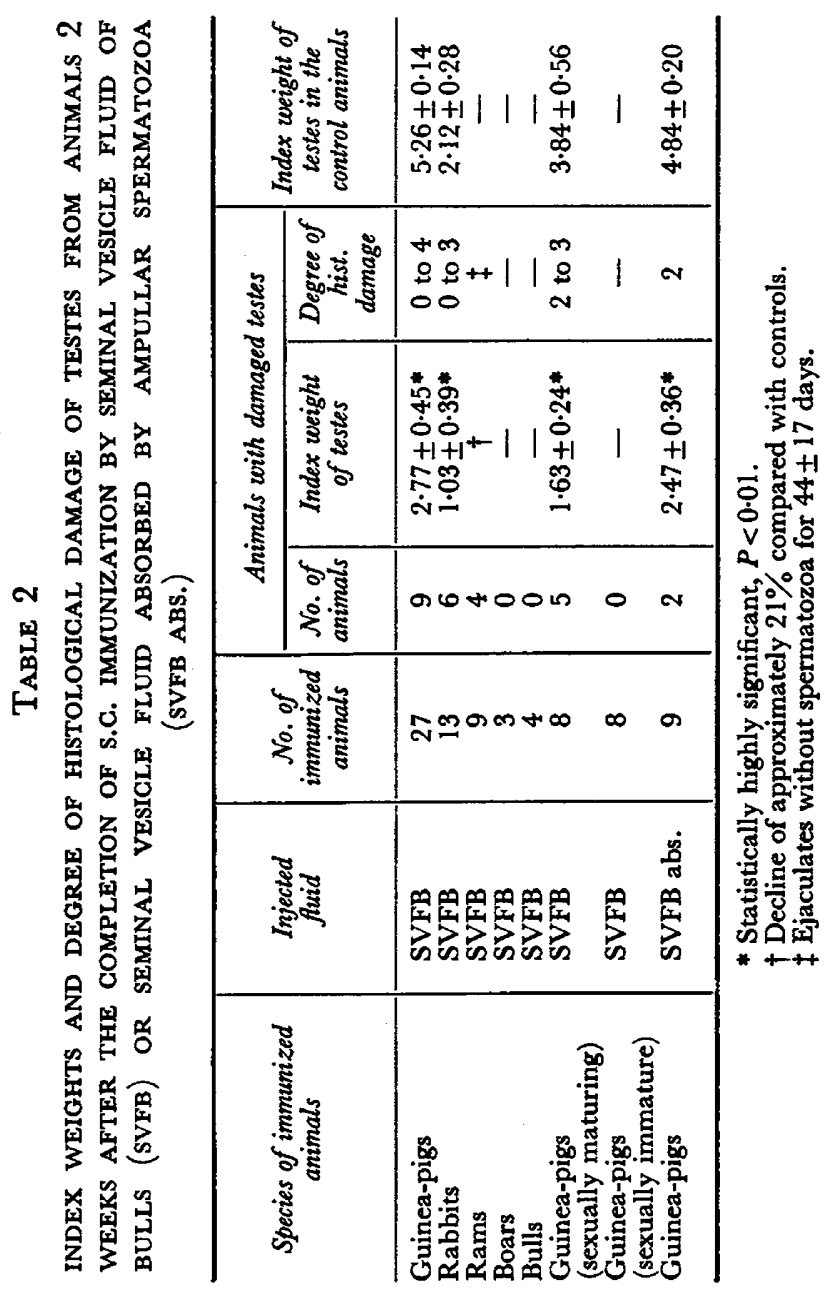


significant changes in their testes. It is interesting that s.c. injections of AFB were without detrimental effect in guinea-pigs although, when administered by intratesticular injection, they caused degenerative changes in the testes of all animals.

None of the fluids injected s.c. into guinea-pigs caused substantial disturbance to the index weight of the seminal vesicles. This observation applied even to seminal vesicle fluid which so strikingly damaged the spermatogenic epithelium of the testis.

\section{Histological changes}

The decrease in testicular weight observed after immunization was accompanied by histological changes. These were marked chiefly by the gradual disappearance of the spermatogenic epithelium from the tubules, and also by a decrease in the number of seminiferous tubules.

The mean diameter of seminiferous tubules in thirty guinea-pigs with normal testes was $155.23 \pm 14 \mu$, but was $119.26 \pm 21.64 \mu$ in thirty guinea-pigs with damaged testes in which the tubules contained only spermatogonia. In nine control rabbits, the mean diameter of the tubules was $163.05 \pm 8.88 \mu$ and in six rabbits with tubules containing only spermatogonia it was $120 \cdot 10 \pm 13 \cdot 16 \mu$. Further damage did not lead to a greater decline in the diameter of tubules for they were infiltrated by interstitial cells and later by connective tissue.

The spermatogenic epithelium showed striking degenerative changes. These involved disorganization of the developing spermatogenic layers, absence of meiotic divisions, meiotic irregularities in the spermatocytes, pycnotic changes in spermatocytes and karyolysis of spermatids. Associated with these changes there was a pronounced decrease and, later, a complete disappearance of intratubular spermatozoa ( $\mathrm{Pl}$. 1, Fig. 1, degree of histological damage =1). Following the disappearance of spermatozoa, the next spermatogenic disturbance observed was the desquamation of spermatogenic layers (histological damage $=1$ to 2 ). In the majority of animals injected s.c., the maximum damage to the testes reached a stage characterized by the disappearance of all spermatogenic cells except the spermatogonia and Sertoli cells (Pl. 1, Fig. 2, histological damage $=3$ ). The degenerative process proceeded to a final phase in those animals which had received intratesticular injections of SVFB and also in some animals which had received s.c. injections of this fluid. Necrobiotic changes were associated with an infiltration of leucocytes and lymphocytes into the seminiferous tubules and the appearance of connective tissue, resulting in

\section{EXPLANATION OF PLATE 1}

Fig. 1. Guinea-pig testicle after s.c. immunization by SVFB. Seminiferous tubules without spermatozoa and spermatids, primary and secondary spermatocytes begin to show degenerative changes. $\times 450$.

FIG. 2. Guinea-pig testicle after s.c. immunization by SVFB. Only degenerated spermatogonia in seminiferous tubules. $\times 450$.

FIG. 3. Guinea-pig testicle injected with SVFB. The basement membrane of tubules is disturbed and the testicle tissue begins to be distorted. $\times 450$.

FIG. 4. Guinea-pig testicle after s.c. immunization by SVFB. Some seminiferous tubules are still normal and the others show degenerative changes. $\times 230$. 
PI..IE:

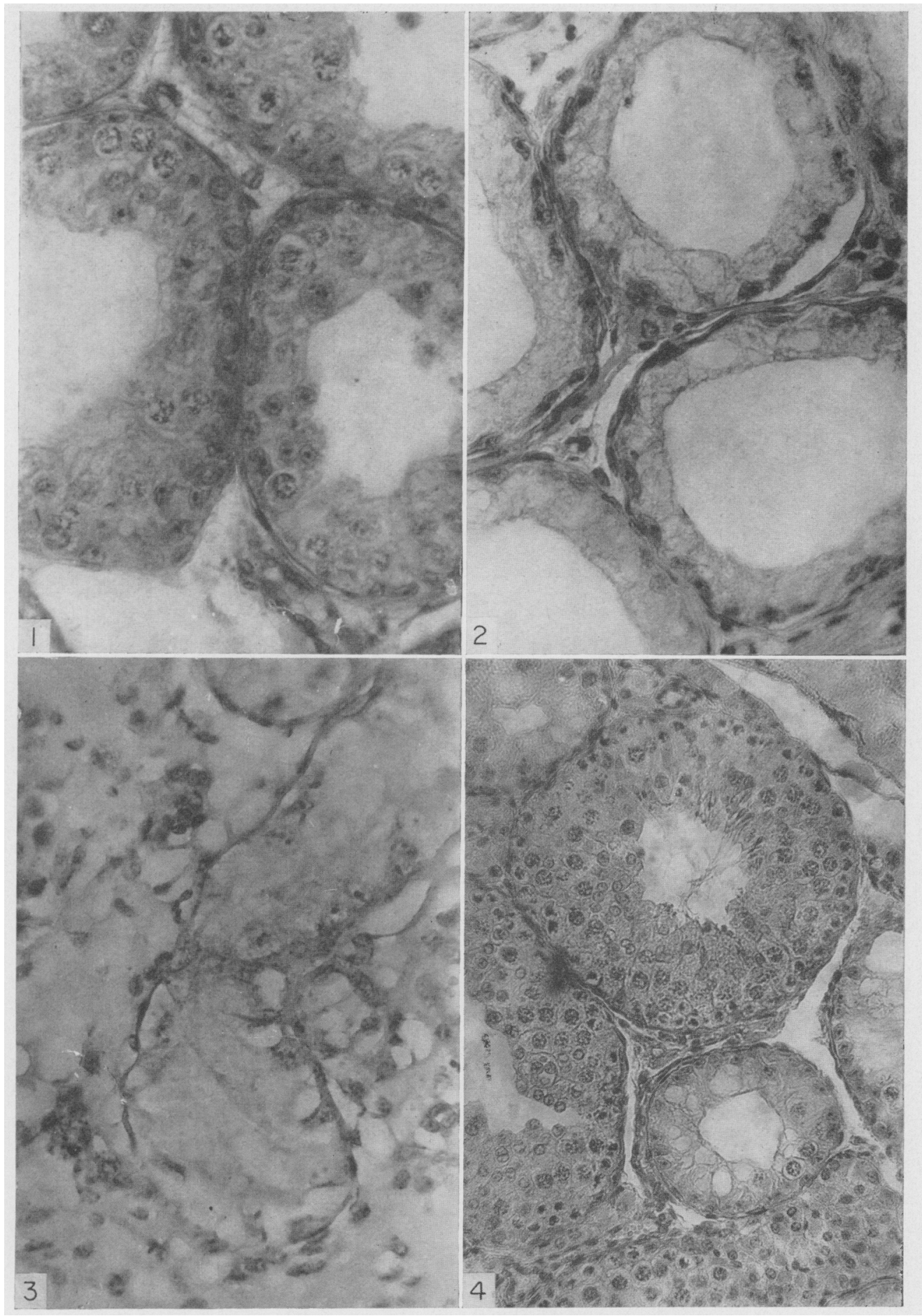

fracing p. 68 : 
the distortion and disappearance of the tubules (Pl. 1, Fig. 3, histological damage $=4$ ).

The degenerative process did not always proceed with equal intensity throughout the testis. Tubules appearing histologically near-normal could be found next to tubules with considerable degeneration of the spermatogenic epithelium (Pl. 1, Fig. 4). Ultimately, the damage usually spread to involve the whole testis.

\section{Demonstration of antibodies}

No antibodies against any of the injected fluids could be detected in the serum of animals which had received one intratesticular injection, using any of the methods described, including the local anaphylaxis test.

In animals injected s.c., however, a considerable number of antibodies could be detected on agar-gel double diffusion plates and by immuno-electrophoresis (Table 3). Ram serum formed four to nine lines on agar-gel and five to nine arcs on immunoelectrophoresis following immunization by SVFB. Antibodies formed against SVFB were directed against proteins of both albumin and globulin type, as shown by immunoelectrophoretic analysis.

There were no differences in number or character of precipitation antibodies between animals with damaged testes and those with normal testes in sheep, guinea-pigs or rabbits.

The agglutination or immobilization of spermatozoa and spermatotoxic antibodies were found only in the sera of males immunized by ampullar and epididymal fluid, and only when using ejaculated bull spermatozoa (Table 3). The titre of spermagglutinins (tail agglutination) ranged from eight to thirtytwo. Cross-reactions between antibodies against SVFB and spermatozoa of sheep and rabbits were not detected.

The study of antibodies observed by complement fixation was impeded by the variable anti-complementary effect of ejaculated, ampullar or epididymal spermatozoa of bulls. The use of SVFB as an antigen was affected by the haemolytic factor present in this fluid.

\section{Further effects of SVFB}

As shown above, intratesticular or s.c. injections of SVFB into sexually mature males cause considerable damage to the testis (Tables 1 and 2; Pl. 1, Fig. 3). Similar treatment of sexually immature rabbit and guinea-pig males did not cause any degenerative changes in their testes (Tables 1 and 2).

Single intratesticular doses and s.c. injections of SVFB caused severe illness in males of all species. For example, nine out of thirty six guinea-pigs injected s.c. with this fluid died during the immunization period. In the remaining males, body-weight dropped by 12 to $31 \%$. Analogous results were obtained with rabbits. In rams, immunization did not result in death, but loss of weight amounted to 6 to $19 \%$. The loss of body-weight, however, was not correlated with degenerative processes in the testes. The other fluids did not cause loss in body-weight.

Degenerative changes caused by SVFB affect only the testicular tissue. The decrease of weight of the epididymides, caused probably by the disappearance 


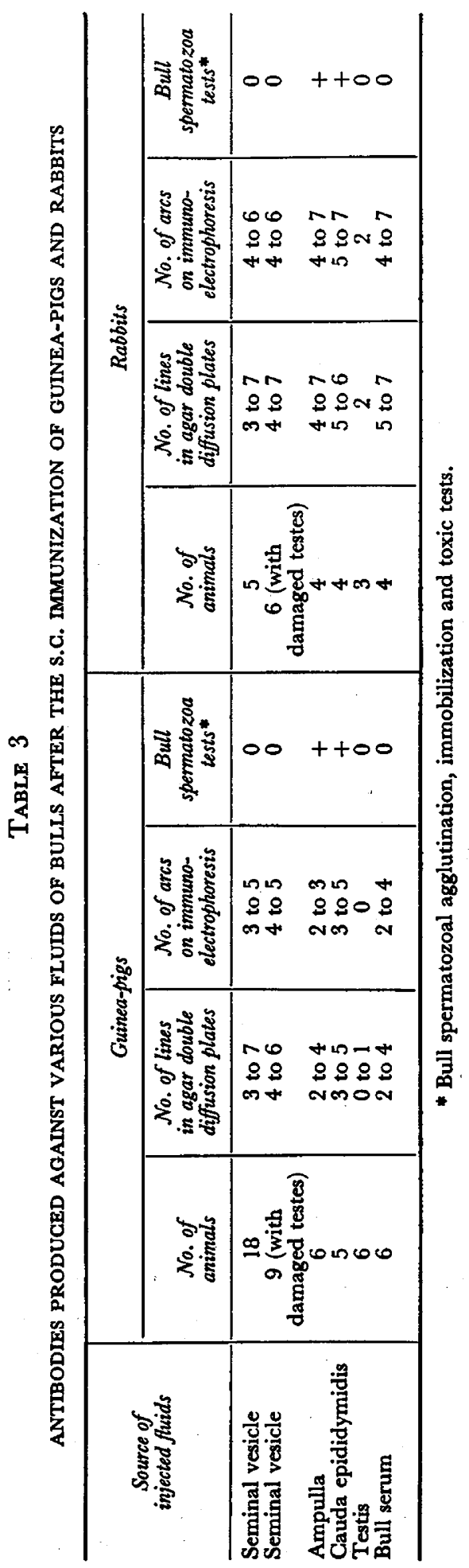


of spermatozoa, is not so pronounced as in the testes. After intratesticular immunization of twelve guinea-pigs, the index weights of the injected testes were $0 \cdot 49 \pm 0 \cdot 10$ and of the uninjected testes $2 \cdot 46 \pm 0 \cdot 27$, respectively, whereas the index weights of cauda epididymidis were $0.34 \pm 0.06$ and $0.50 \pm 0.07$, respectively.

\section{DISCUSSION}

The most intensive damage to testicular tissue was induced by fluid from the seminal vesicles. The changes resulting from the intratesticular administration of this fluid could not be accounted for on the basis of its haemolytic property and ensuing auto-immune response since the same effect resulted even after removal of haemolysins by absorption with ampullar spermatozoa. Physical damage to the testes by intratesticular injections also seems to be out of the question since other fluids administered in the same way and in the same volume did not cause degeneration. Further evidence for a specific effect of SVFB was given by the damage to testes of animals injected subcutaneously.

The testicular changes may be due to the reaction of antibodies with closely related antigens in SVFB and in some cells of the spermatogenic tissue. Histological analyses of testes from animals injected s.c. showed that the principal damage was incurred by spermatids and spermatocytes, the spermatogonia and Sertoli cells being unaffected. In this context, it is worth noting that, in sexually immature rabbits where only spermatogonia were present at the time of injection, the testes showed no degenerative changes after immunization by SVFB.

The intratubular spermatozoa showed no morphological damage. Their decrease in number or complete absence from seminiferous tubules, epididymides, and ram ejaculates appeared to be the result of disturbance to their cell-precursors. The failure of antisera obtained from males with damaged testes to agglutinate, immobilize or be toxic to ejaculated spermatozoa lends confirmation to this suggestion. In the intertubular tissue, the interstitial cells seemed to be the most resistant to the treatment, and these cells appeared to colonize areas left by the shrinkage of the seminiferous tubules. Only in the severest cases of degeneration, were the interstitial tissues also damaged. Degenerative changes elicited by SVFB resembled the changes caused by administration of bull hyaluronidase, i.e. spermatozoal antigen, to male rabbits (Yamane, 1956). If the spermatogenic effect of SVFB is due to an immunological reaction, then the common or cross-reacting antigen in SVFB and in spermatogenic tissue is not species-specific.

Questions arise concerning the site of antibody formation and its localization and binding to the SVFB antigen and to the corresponding testicular antigen. The existence of a certain barrier, which blocks circulating antibodies before they can reach the seminiferous epithelium, was indicated by the observation that more than half of the animals immunized with circulating antibodies did not have damaged testes, and that some animals immunized s.c. with SVFB had one damaged testis while the other was intact. Alternatively, some antibodies may not be able to invoke degenerative changes. Binding of serum antibodies to homologous or heterologous spermatozoa was not detected, and it was not possible, by means of antisera from males with damaged testes, to 
block the SVFB substance responsible for causing the degeneration. Besides circulating antibodies, other antibodies might be formed locally especially in animals given intratesticular injections. Immunization s.c. may also result in local antibody formation due to the distribution of the antigen to the testis by lymphocytic cells.

It seems that immune antibodies, once established in the organism, have a protective effect on the testicular epithelium. Secondary immunization of rams whose testes had already been damaged by previous immunization did not appear to cause further damage. Analogous results were obtained in experiments on bulls (Matoušek et al., 1966).

Existing preliminary data indicate that SVFB does not affect homologous testicular tissue, for none of four bulls immunized for 4 weeks showed any testicular damage. More prolonged treatment with SVFB might be required, since other work with long-term administration of seminal vesicle fluid of boars to homologous males indicated that the length of the period of immunization might be important for the development of the iso-immune process. Future experiments will be designed to elucidate the antigenic cross-reactions between SVFB and rabbit or guinea-pig testes (cross-reactions between this fluid and ram or rabbit spermatozoa were negative) and any toxic effect of SVFB on spermatogenesis.

\section{ACKNOWLEDGMENTS}

I would like to thank my collaborator Mrs Vackova who helped me during the laboratory tests.

\section{REFERENCES}

Campbell, D. H., Garvey, J. S., Gremer, N. E. \& Sussdorf, D. H. (1963) Methods in immunology, pp. 215-216. Benjamin, New York.

Fulka, J., Valenta, M., Pavlok, A. \& Icha, F. (1960) Diferenciace živých a mrtvých spermií cyaninem B. ŻivoZǐñ výroba, 5, 905.

Grabar, P. \& Burtin, P. (1964) Immuno-electrophoretic analysis, pp. 3-29. Elsevier, Amsterdam.

KWAPINSK, J. B. (1965) Methods of serological research, pp. 276-287. Wiley, New York.

Matoušex, J. (1966) Antigenicity of the seminal vesicle fluid in bulls. Xth Int. Congr. Anim. Blood Grps., Paris, p. 513.

Matoušek, J., Dostal, J. \& FulKa, J. (1966) Antigenicity and polymorphism of the seminal vesicle fluid in boars. Xth Int. Congr. Anim. Blood Grps, Paris, p. 523.

QuinuIvan, W. L. G. (1966) Antigen-antibody reactions with human semen. Fert. Steril. 17, 722.

YAMANE, J. (1956) The role of testicular hyaluronidase in spermatogenesis and fertilization. Proc. 2nd Wld Congr. Fert. Steril., Naples, 2, 1080. 